\title{
Investigation on Standardization of Modal Space by Ratio for MDOF Micro-Macro Bilateral Teleoperation Control System
}

\author{
Lee Jun Wei ${ }^{1}$, Ahmad Zaki bin Hj Shukor ${ }^{1} \&$ Muhammad Herman bin Jamaluddin ${ }^{1}$ \\ ${ }^{1}$ Center for Robotic and Industrial Automation (CeRIA), Faculty of Electrical Engineering, Universiti Teknikal \\ Malaysia Melaka, Durian Tunggal, Malaysia \\ Correspondence: Lee Jun Wei, Faculty of Electrical Engineering, Universiti Teknikal Malaysia Melaka, Durian \\ Tunggal, Malaysia. Tel: 6013-632-8128. E-mail: junwei1690@gmail.com
}

Received: May 16, 2016

doi:10.5539/mas.v10n11p98
Accepted: June 20, 2016

Online Published: July 29, 2016

URL: http://dx.doi.org/10.5539/mas.v10n11p98

\begin{abstract}
This paper presents the research on micro-macro bilateral teleoperation control system of two ink planar manipulator. The micro-macro bilateral control system consists of different size between master and slave system using geared DC-motor. Both master and slave manipulators are actuated by DC-Micromotor attached to planetary gearhead to increase the output torque. In the previous researches the most common actuators used were linear motor and direct-drive DC motors. However, the application of DC motor with gearhead are vast in industry, which need high output force or torque. Thus in this paper, research on micro-macro bilateral teleoperation control system is proposed with the use of gear with the DC-motor. The micro-macro bilateral teleoperation control system provides the human operator with a sense of feel to a micro or macro environment as if it is in the same scale environment. Thus a standardization method is proposed to achieve micro-macro bilateral teleoperation control system. During the experiment, experiment on free motion and contact motion are conducted to validate the proposed setup in bilateral teleoperation control system. The position and torque responses of both master and slave manipulators are observed. The operationality and reproducibility of this proposed system are evaluated through proposed experimental results.
\end{abstract}

Keywords: micro-macro, standardization, modal space, haptics, MDOF bilateral teleoperation control system, geared DC-motor, operationality, reproducibility

\section{Introduction}

Further application of bilateral control system is micro-macro bilateral teleoperation control system. The micro-macro bilateral teleoperation control system consists of macro master system and micro slave system or vice versa. To manipulate macro object, the slave system is generally large compared to master system. This is where the scaling technique is used for force and position between master and slave manipulator which is different in size. For example, to manipulate micro object, the slave system is smaller while master system is the size of human operator. This method enables human operator to manipulate the master and slave system with a different size structure. The micro-macro bilateral teleoperation control system provides the human operator with a sense of feel to a micro or macro environment as if it is in the same scale environment. In other words, the human operator feels the reaction force as if they are touching the real micro or macro environment. It is effective for manipulation which cannot be operated directly. Micro-macro bilateral teleoperation system provides benefit in many fields such as industrial environment and medical applications. A micro-macro bilateral teleoperation is essential in medical field such as minimally invasive surgery (MIS) for brain surgery (Salcudean \& Yan, 1994).

Since the micro-macro bilateral teleoperation control system consists of different size between master and slave system, the inertia and scaling of the master and slave system manipulators are different. This also leads to force and position scaling required for micro-macro bilateral teleoperation control system. Few researches about micro-macro bilateral teleoperation control system on control method have been presented. K. Kaneko presented micro-macro bilateral teleoperation control system based on operational force feedforward (Kaneko et al., 1998). K. Takeo proposed an alternative control algorithm for micro-macro teleoperation system (Takeo \& Kosuge, 1997). A. Sano proposed stabilization method on bilateral teleoperation control system with scaling factors based on $H_{\infty}$ theory (Sano et al., 2000). Then the standardization between master response and slave response by 
nominal mass of master and slave system in micro-macro bilateral teleoperation control was proposed (Shimono \& Ohnishi, 2007; Susa et al., 2007). S. Susa presented scaling factors of the control gains at master and slave system in the micro-macro bilateral teleoperation control system (Susa et al., 2008). S. Susa further presented with three channel micro-macro bilateral teleoperation control with arbitrary scaling factors able to achieve high accuracy control although using lesser information channels (Susa et al., 2008). N. Motoi proposed a modal space disturbance observer (MSDOB) in the micro-macro bilateral teleoperation control system to realize high transparency (Motoi et al., 2010).

In particular, linear motor is often utilised in this field of research. It is powerful, but cost is high. Linear motor provides very high linear force along its length. However, linear motor cannot provide rotary motion for applications such as in articulated robotic arm. Moreover, the DC-motor has limitation of torque output generated by the specific size of the motor. To obtain higher torque, the size of motor will need to be increased, which is not practical for haptic applications. To do so, the most desirable way is by attaching gears. Moreover, the applications of geared DC-motor are vast in industry due to the need for high output torque.

Until now, micro-macro bilateral teleoperation control system for MDOF systems using geared DC-motor has not been derived yet. This is significantly useful in robotic surgery because it requires MDOF. Thus, our research focuses on two link rotary planar manipulator for micro-macro bilateral teleoperation control system. Nevertheless, transmission of haptic information is used with micro-macro bilateral teleoperation control system with the use of geared DC-motor drives. The micro-macro bilateral teleoperation system uses the planetary geared DC-motor to achieve a realization of high torque and small size motor for haptic applications. Since most of the researchers researched in the bilateral teleoperation control system by using linear motor and direct-drive DC motors, this proposed setup is to give an idea in the application of robotics such as in industries which mostly uses geared motor. The performance of the proposed setup is analysed by using reproducibility and operationality.

Even though teleoperation systems used for human to operate larger scale slave manipulator such as excavator, Autonomous Underwater Vehicle (AUV) for exploration or maintenance purposes, these applications have no haptic feedback information from the environment. To execute meaningful task remotely, the operator is able to simultaneously control the slave manipulator and efficiently obtain the information from the environment side. This is to say that the operator's task execution performance is improved due to haptic feedback.

This paper is organized as follows. The first part introduces about the bilateral teleoperation control system following by the workspace observer (WOB) and reaction force observer (RFOB) for two DOF planar bilateral teleoperation manipulator. Then, the proposed method of standardization of modal space by ratio for mdof micro-macro bilateral teleoperation control system is introduced. Next the experimental setup and experimental manipulations is demonstrated. At the result and discussion, the experiments of free and contact motions are conducted and discussed. Ultimately, this paper focused on the performance of the proposed setup is analysed by using reproducibility and operationality.

\section{Bilateral Teleoperation Control System}

In bilateral teleoperation control system, the information from the remote environment is acquired to help human operator to feel as if they are physically present at the remote place. While the human operator moves the master manipulator, its position responses transmit to the slave manipulator which causes it to follow every master manipulator's movement. When the slave manipulator contacts an object at a remote environment, the object reaction force responses transmit back to the human operator to sense the reaction force of the object. The idea of this bilateral teleoperation system is that the position and force tracking between master and slave system are followed at every time instant. Moreover, the position and force tracking at master and slave system are matched in steady state, which can lead to stable and transparent teleoperation system (Lawrence, 1993). This ideal behavior of teleoperation bilateral system enables the human operator to feel they are directly interacting with the object at a remote environment.

In the application of bilateral control system, only position information from master and slave manipulators are required. The position information from master system is transmitted to the slave system then the slave system will try to follow every command of position information from master system and vice versa. This control scheme is completely symmetric. The master and slave manipulators are followed closely to each other if the position controller have good position tracking capabilities.

In order to fulfill the bilateral control system requirement and to comply the concept in a haptic system, this bilateral control system is equipped with disturbance observer (DOB) (Ohnishi et al., 1994) and the reaction force observer (RFOB) (Murakami \& Ohnishi, 1993). The differential mode of the system is position controlled 
using DOB while the common mode of the system is force controlled using RFOB. This system can automatically calculate force disturbance and external force that present in both master and slave system. The sensorless type of motion control system provide robustness of the system. Thus, the operator at the master system can feel the real sensation of the environment at the slave system even though the operator is not at the environment area. Figure 1 shows the block diagram of single link bilateral control based on acceleration control.

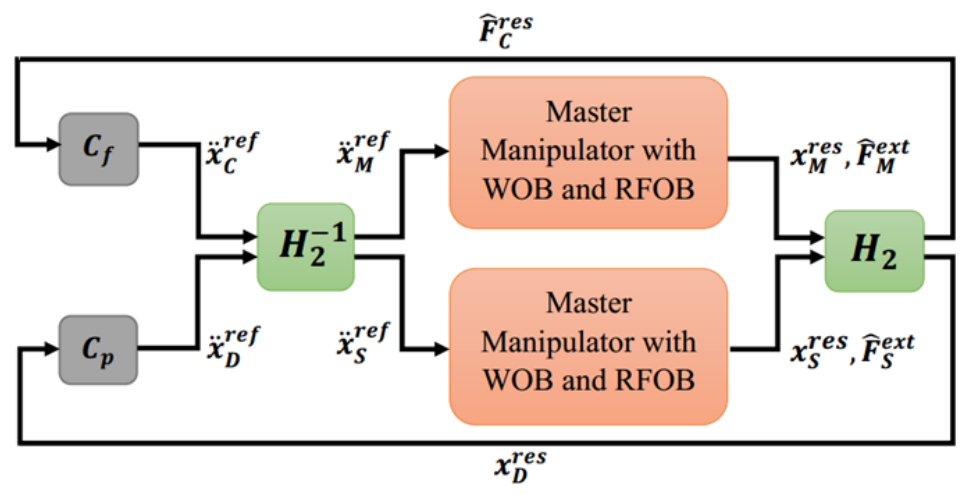

Figure 1. General acceleration based four channel bilateral controller

\subsection{Disturbance Observer (DOB) and Reaction Force Observer (RFOB)}

In the previous paper, the implementation of workspace observer into Direct Cartesian scheme was presented and results showed improvement in the workspace control (Wei et al., 2015). Although bilateral control system is used in this paper, the meaning and implementation of WOB are the same. However, reaction force observer (RFOB) is used too.

The DOB is a robust control tool that able to estimate the external disturbance and system uncertainties. DOB is also designed to cancel the disturbance torque as quickly as possible which act as disturbance compensation in a motion control system. Robust motion control is attained by using the disturbance observer, the robust motion controller makes a motion system to be an acceleration control system (Katsura et al., 2007). That is the reason DOB is implemented in order to establish robust acceleration controller (Ohnishi et al., 1996). The output of DOB is the friction effect under the constant angular velocity motion in the mechanism. A robust system means that the system is insensitive to the external disturbance and parameter variations and will maintain control. It can obtain wider bandwidth than force sensor due to settling sampling time and observer gain by using DOB (Murakami \& Ohnishi, 1993).

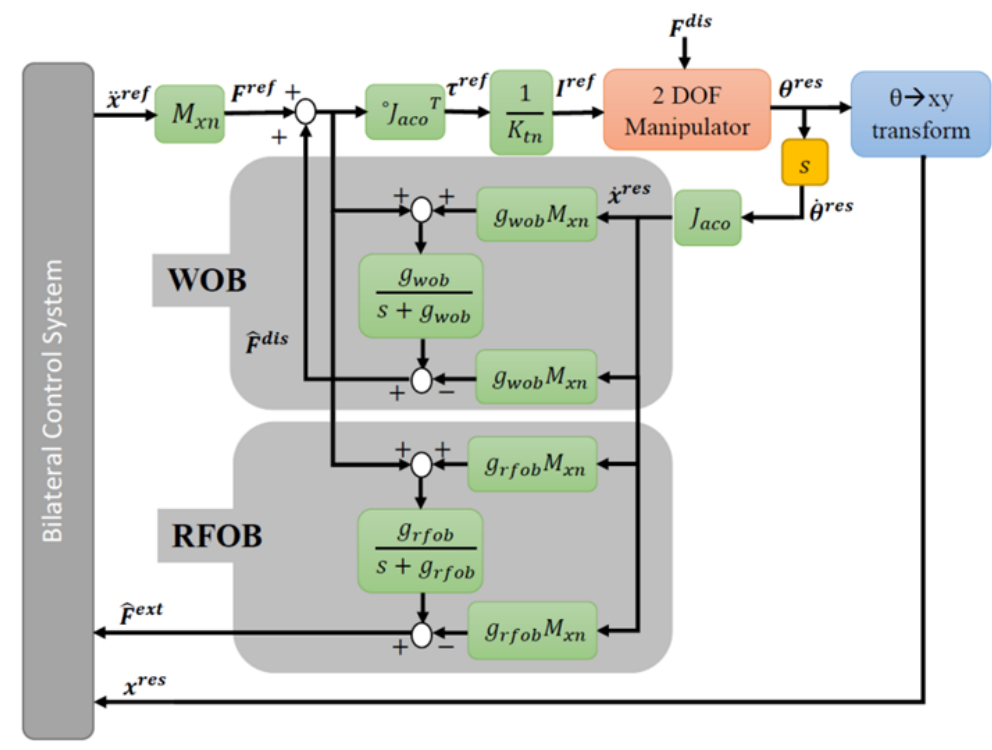

Figure 2. Block diagram of workspace based disturbance observer and reaction force observer 
Figure 2 shows the block diagram of the implementation of WOB and RFOB into the MDOF bilateral control system. The estimated disturbance, $\hat{F}^{\text {dis }}$ and estimated external force, $\hat{F}^{\text {ext }}$ for master and slave system are estimated using WOB and RFOB for both $x$ axis and $y$ axis, respectively.

However, identification of frictional force is needed in advance to estimate the external torque. The friction disturbance can be compensated by DOB only if an accurate friction model is provided. Here, the frictional force is not compensated and cannot be ignored. Thus later in the experiments, the system has friction effects largely from planetary gear. The friction force contributed as an operational force. However, the compensation of friction force is not in the scope.

\subsection{Standardization of Modal Space by Ratio for MDOF Micro-Macro Bilateral Teleoperation Control System}

In MDOF bilateral system with different structure, the operational range and mass between master and slave system are different. For instances, both system can have completely same motion but different operational range. Similarly, the force is reproduced much more than the other one if one system is much bigger than the other. Thus, the position and force between both systems need to be standardized by scaling second order quarry matrix;

$$
H_{2}=\left[\begin{array}{cc}
1 & \alpha \\
1 & -\beta
\end{array}\right]
$$

where $\alpha$ is the scaling ratio of force information and $\beta$ is scaling ratio of position information. Thus, the slave system reproduces force and track position based on $\alpha$ and $\beta$ gain with respect to master system. These scaling enables bilateral teleoperation with arbitrary ratio.

$$
\left[\begin{array}{cc}
F_{C}^{r e s} & * \\
* & x_{D}^{r e s}
\end{array}\right]=\left[\begin{array}{cc}
1 & \alpha \\
1 & -\beta
\end{array}\right]\left[\begin{array}{ll}
\hat{F}_{M}^{\text {ext }} & x_{M}^{r e s} \\
\hat{F}_{S}^{\text {ext }} & x_{S}^{r e s}
\end{array}\right]=H_{2}\left[\begin{array}{ll}
\widehat{F}_{M}^{e x t} & x_{M}^{r e s} \\
\widehat{F}_{S}^{\text {ext }} & x_{S}^{r e s}
\end{array}\right]
$$

Force information is scaled by the equivalent nominal mass matrix $M_{x x n}$ and $M_{y y n}$ in each system. The force scaling ratio, $\alpha_{x}$ and $\alpha_{y}$ are designed to scale force at each axis of $\hat{F}_{S}^{\text {ext }}$ of $x$ and $y$ axis as shown in Equation (2.3) and Equation (2.4), respectively.

$$
\begin{gathered}
\alpha_{x}=\frac{M_{x x M n}}{M_{x x S n}} \\
\alpha_{y}=\frac{M_{y y M n}}{M_{y y S n}}
\end{gathered}
$$

Position information is scaled by the operable region of each system by $l_{1}$ and $l_{2}$. The position scaling ratio, $\beta$ is designed by utilizing nominal mass $l_{1}$ and $l_{2}$ as:

$$
\beta=\frac{l_{M 1}}{l_{S 1}}=\frac{l_{M 2}}{l_{S 2}}
$$

Figure 3 shows the proposed four channel micro-macro bilateral control system with respect to the standardized modal space.

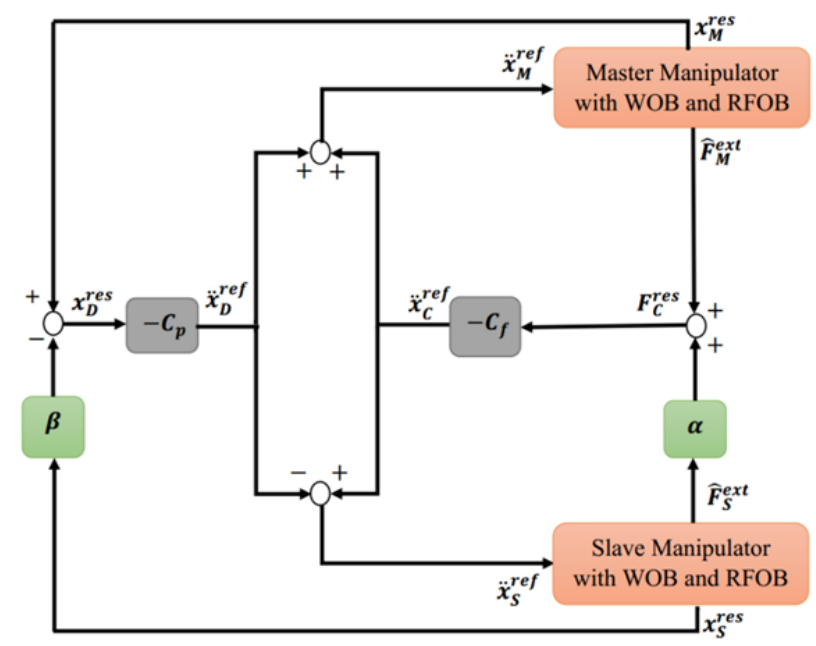

Figure 3. Proposed micro-macro bilateral control system 


\subsection{Hardware Setup}

There are two sets of two link planar manipulators. Each joint is actuated by a planetary geared DC-Micromotor with incremental encoder. It has 5000ppr (pulse per revolution) before gearhead. The links are designed with a $0.13 \mathrm{~m}$ each with a base attached to a platform to prevent any unwanted vibration. The link can either be used to operate the system by human operator on master side or to the environmental contact on the slave side. The links at both master and slave side are in horizontal orientation that produces zero gravitational motion. Thus, only disturbance effect and frictional force presented in the gearhead and motor. Moreover, the main purpose of this setup is to investigate the operationality torque and reproducibility of this propose geared micro-macro bilateral teleoperation control system.

Planetary gearhead able to provide higher torque for a low torque DC-Mircomotor. Moreover, the backlash is crucial to the haptic application where it can affect the performance of the bilateral system. The output of master and slave manipulator is position that measured by the encoders mounted at the back of each motor shaft. The velocity response is obtained by derivative of position response and Low Pass Filter (LPF) filters away noise from the signal in the control loop within the computer software. Within the Simulink, the processed data is set to analogue voltage reference signal from the Micro-Box to the motor driver. Motors are driven by Maxon motor driver (ESCON 50/5) in current based control mode. The reference value represents the desired current that the motor driver injected to the motor. The motor torque is directly proportional to the motor current (Hace \& Jezernik, 2010). Figure 4 shows the experimental setup of this research.

Nevertheless, before conducting the experiment, the position controller gains, force controller gain, bandwidth of DOB and RFOB/RTOB are stochastically tuned in order to achieve the required performance of the particular system. These gains are tuned based on random trials until the overall system is stable and shows good performancev (Wei et al., 2016).

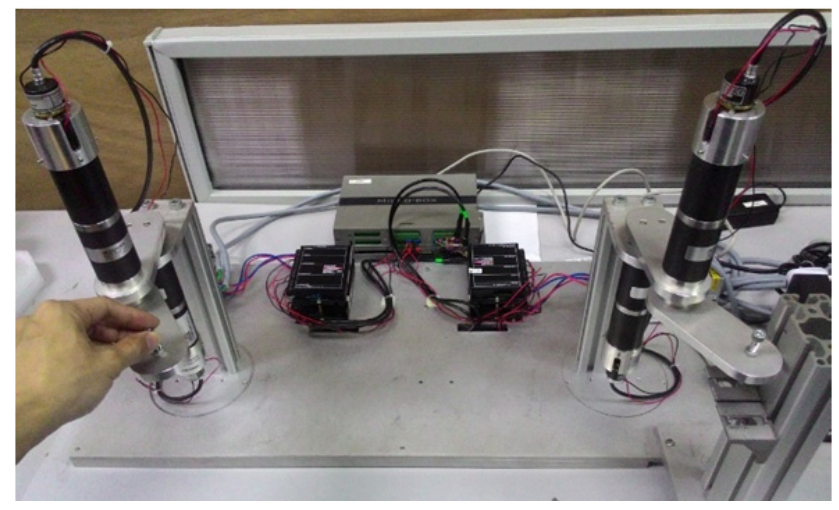

Figure 4. Experimental Setup

\subsection{Experimental Manipulations}

In this part, there are two experiments to be conducted. The first experiment is free motion and the second experiment is contact motion. During the free motion, the human operator control freely at master system while the slave system also follow freely without contact with any obstacle. During the contact motion, the initial position of the end-effector of the slave system is near to the surface of the objects as shown in Figure 4. The human operator operates the master manipulator manually in order for the slave manipulator to contact the object. During contact motion, human operator holds the master handle and move the handle while the slave handle is constrained by a static object. The human operator then applies torque at the master handle. The hard object is an aluminium block while the soft object is a sponge. Particularly, the human operator applied force on $y$ axis at the end-effector of master manipulator during contact motion.

The force and position response from both master and slave system are recorded, for both free motion and contact motion experiments. The position response from master and slave system are obtained from rotary encoder while the external force applied to the master and slave system are estimated by RFOB. The force and position response from both master and slave system are compared with each other to validate the operationality and reproducibility of the bilateral control system. Nevertheless, the operationality can be validated from the free motion experiment while the reproducibility can be validated from the contact motion experiment. Then, the evaluation of this part of experiment is explained in discussion and conclusion.

Here, singularity is not cancelled. For this case, the singularity points according to end-effector are when the 
second joint (elbow) is at $0^{\circ}$ or $180^{\circ}$.

\section{Results}

In this part of the experiment, proposed micro-macro bilateral teleoperation control system is applied to two link planar manipulator master and slave system. In this case, this is a scaled up bilateral teleoperation control system, where the master system is micro while the slave system is macro. It is noted that both master and slave manipulator has the same structure, but the gear reduction ratio in master manipulator, $N_{M 1}: N_{M 2}$ is relatively smaller than gear reduction ratio in slave manipulator, $N_{S 1}: N_{S 2}$. This lead to each joint at master manipulator has lower torque constant compared to slave manipulator. Similarly, the pulse per revolution was also affected by the gear reduction ratio. This lead to different pulse per revolution at joint between master and slave system. Moreover, in order to perform a micro-macro bilateral teleoperation control system, the master system has two times virtually smaller nominal mass $M_{n 1}$ and $M_{n 2}$ than the slave system. In this case, $M_{x x n}$ and $M_{y y n}$ are different between master and slave system. It is also noted that slave system is virtually two times the operable range compared to master system, this master- slave system has the same operable area, though. This means the length of each link, $l_{S 1}$ and $l_{S 2}$ at slave manipulator is two times compared to master system. The experiments are conducted with the parameters shown in Table 1.

Table 1. Parameters in Experiment

\begin{tabular}{clc}
\hline Parameter & Description & Value \\
\hline$l_{M 1}$ & Master Link 1 & $0.13 \mathrm{~m}$ \\
$l_{M 2}$ & Master Link 2 & $0.13 \mathrm{~m}$ \\
$l_{S 1}$ & Slave Link 1 (Virtual) & $0.26 \mathrm{~m}$ \\
$l_{S 2}$ & Slave Link 2 (Virtual) & $0.26 \mathrm{~m}$ \\
$\beta$ & Position scaling factor & 0.5 \\
$M_{M n 1}$ & Master nominal mass 1 & $1.085 \mathrm{~kg}$ \\
$M_{M n 2}$ & Master nominal mass 2 & $0.19 \mathrm{~kg}$ \\
$M_{S n 1}$ & Slave nominal mass 1 (Virtual) & $2.17 \mathrm{~g}$ \\
$M_{S n 2}$ & Slave nominal mass 2 (Virtual) & $0.38 \mathrm{~kg}$ \\
$\alpha$ & Mass scaling factor & $\approx 0.5$ \\
$K_{t M n}$ & Master nominal torque constant & $0.3184 \mathrm{Nm} / \mathrm{A}$ \\
$K_{t S n}$ & Slave nominal torque constant & $0.7164 \mathrm{Nm} / \mathrm{A}$ \\
$K_{p}$ & Position gain & 3000 \\
$K_{d}$ & Velocity gain & 110 \\
$K_{f}$ & Force gain & 2 \\
$N_{M 1}: N_{M 2}$ & Master gear ratio & $16: 1$ \\
$N_{S 1}: N_{S 2}$ & Slave gear ratio & $36: 1$ \\
$g_{d o b}$ & Cut-off frequency of disturbance observer & $50 \mathrm{rad} / \mathrm{s}$ \\
$g_{r t o b}$ & Cut-off frequency of reaction force observer & $50 \mathrm{rad} / \mathrm{s}$ \\
\hline
\end{tabular}

\subsection{During Free Motion}

In this section, the free motion experiment is conducted. Free motion means that human operator manipulates the master manipulator freely and the slave manipulator doesn't contact anything. This experiment is to investigate the operational torque of this bilateral teleoperation control system during free motion. Operationality is degree of operational force which human operator feels besides reaction force from the environment desired for comfortable operation for human operator (Iida \& Ohnishi, 2004). Figure 5 shows free motion experiment result while Figure 6 shows the XY trajectory response during free motion (noted that the graph shows the origin of master and slave manipulator are at the same local frame). 

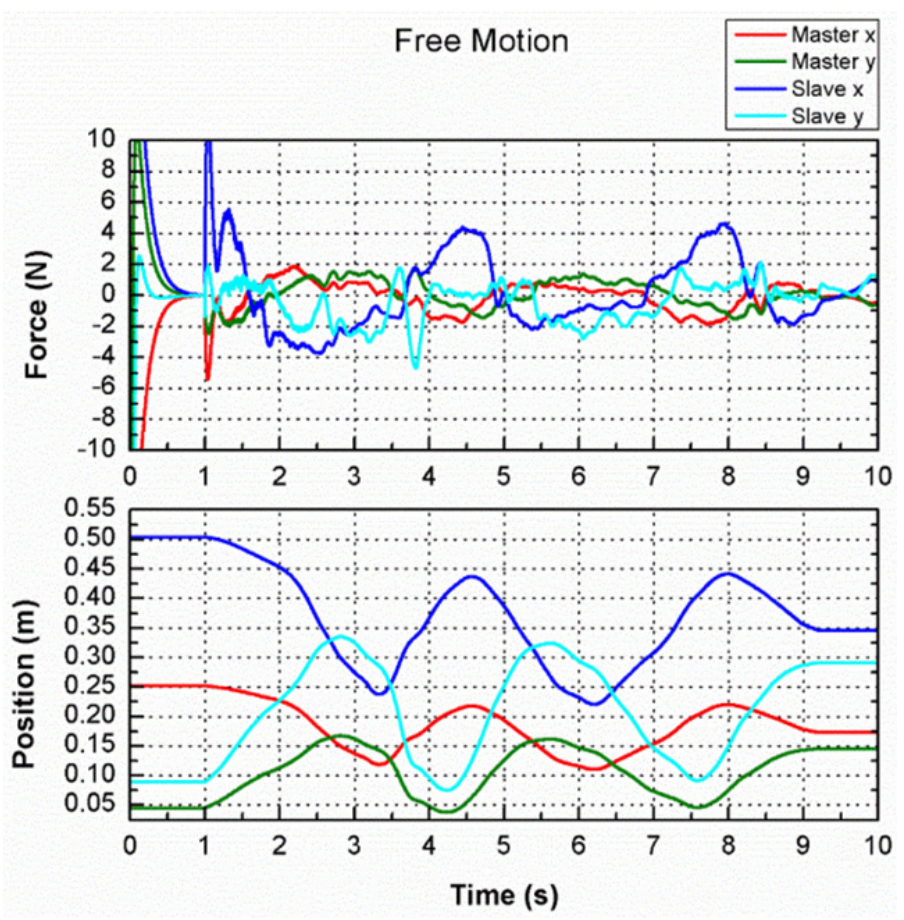

Figure 5. Force and position response during free motion

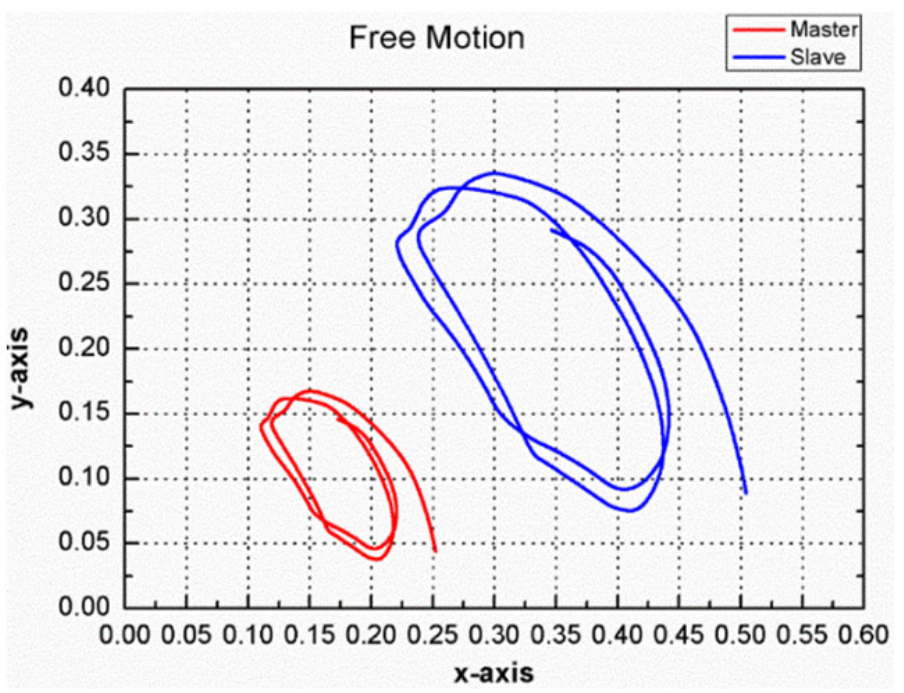

Figure 6. XY trajectory response during free motion

During the free motion, human operator was able to move the master manipulator freely as shown in Figure 5 Because the master manipulator has lower gear reduction ratio, the frictional force is low, thus the operational force is very low compared to slave manipulator which has higher gear reduction ratio. Human operator operated the master manipulator with ease. Nevertheless, the force for both $x$ and $y$ position tracked at slave system is twice larger force than master manipulator. This is due to the virtual mass at slave system set was twice larger than master system. Overall, the law of action and reaction are achieved between master and slave system.

Whereas, the $x$ and $y$ position response from slave manipulator are two times longer than the master manipulator for both $x$ and $y$ position as shown in Figure 5, Figure 6 shows the position response of XY trajectory for both master and slave manipulator during random free motion, where the slave manipulator has virtually twice operation range compared to master manipulator. Again, the position response from both master and slave system are tracked perfectly as the trajectory of both master and slave system are the same. 


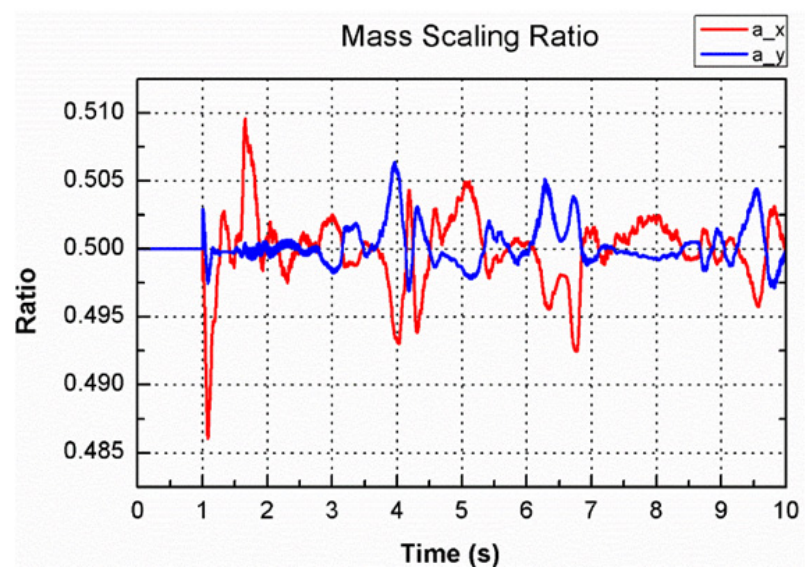

Figure 7. Scaling ratio of $\alpha_{x}$ and $\alpha_{y}$

Figure 7 shows the scaling ration of $\alpha_{x}$ and $\alpha_{y}$. Since the $\mathrm{M}_{1}$ and $\mathrm{M}_{2}$ between slave system is virtually twice larger than the master system, the nominal equivalent mass matrix, $M_{x x n}$ and $M_{y y n}$ between master and system were also affected. Furthermore, the $\alpha_{x}$ and $\alpha_{y}$.changes its scaling ratio as the posture of the two link manipulator changes. After all, the ratio deviated around 0.5 scaling gain of $\alpha_{x}$ and $\alpha_{y}$ as shown in Equation 2.3 and Equation 2.4, respectively.

\subsection{During Contact Motion}

In this section, the contact motion experiment is conducted. The human operator makes three contact motions with the object within 10s. This experiment is to investigate the reproducibility of this micro-macro bilateral teleoperation control system when it comes to contact with an object. Reproducibility is the degree of reproduction of environmental impedance in master side which is the fundamental motive in bilateral teleoperation control system (Iida \& Ohnishi, 2004). According to Figure 8 and Figure 9, contact motion experiment on aluminium (hard object) and sponge (soft object) are operated manually by human operator, respectively. Figure 10 and Figure 11 shows the XY trajectory response during contact motion on aluminium (hard object) and sponge (soft object) are operated manually by human operator, respectively (note that the graph shows the origin of master and slave manipulator at the same local frame).
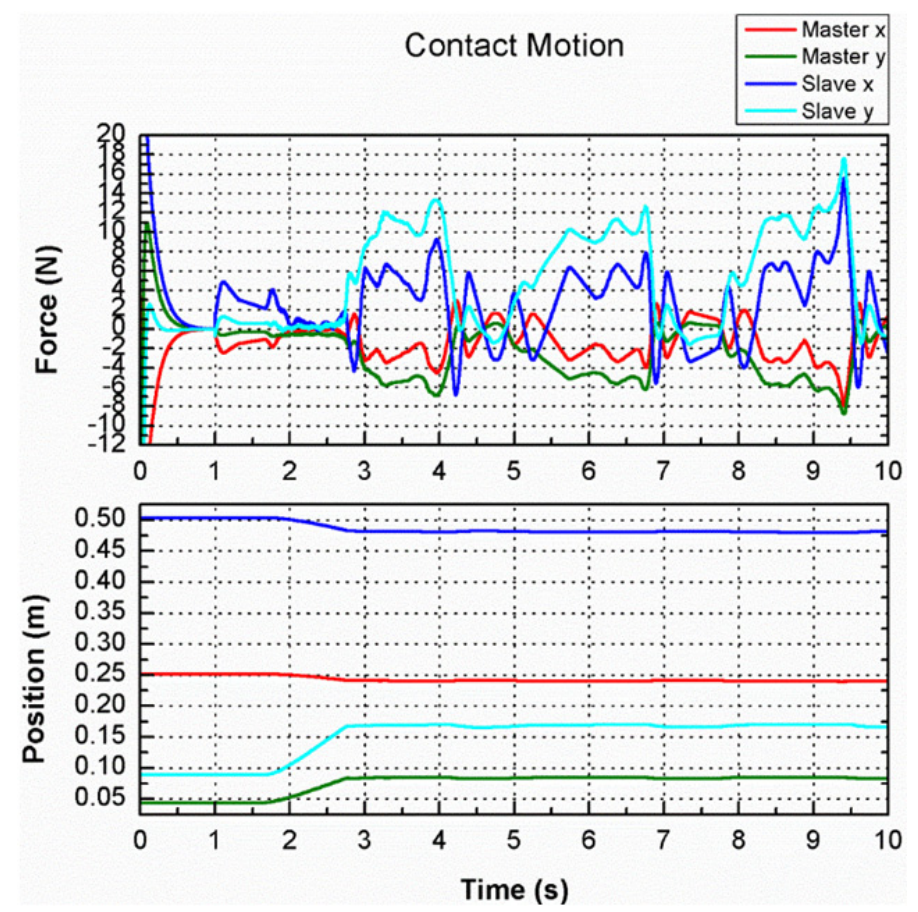

Figure 8. Force and position response during contact motion (hard object) 


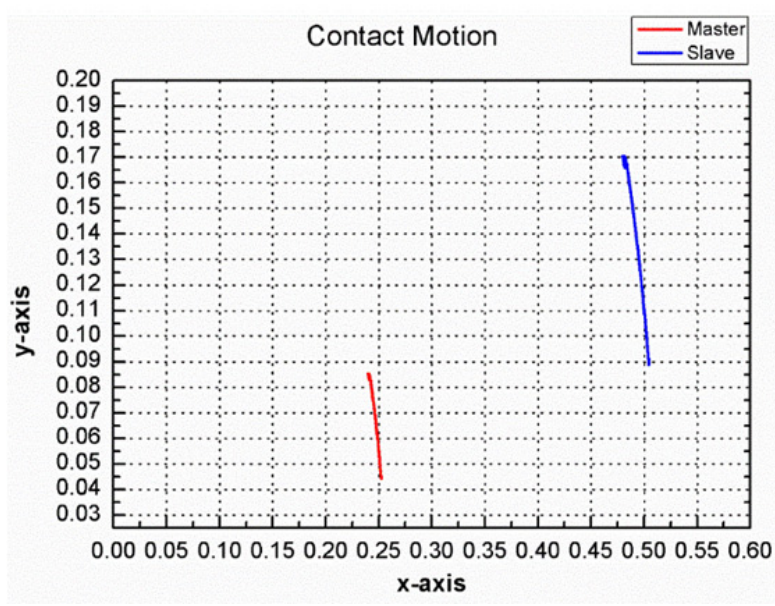

Figure 9. XY trajectory response during contact motion (hard object)
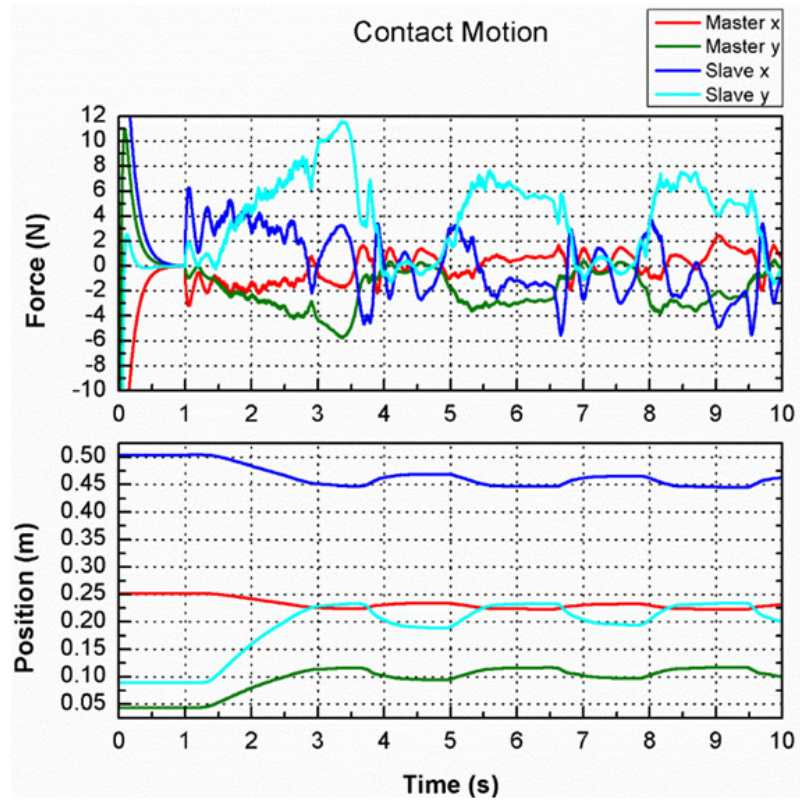

Figure 10. Force and position response during contact motion (soft object)

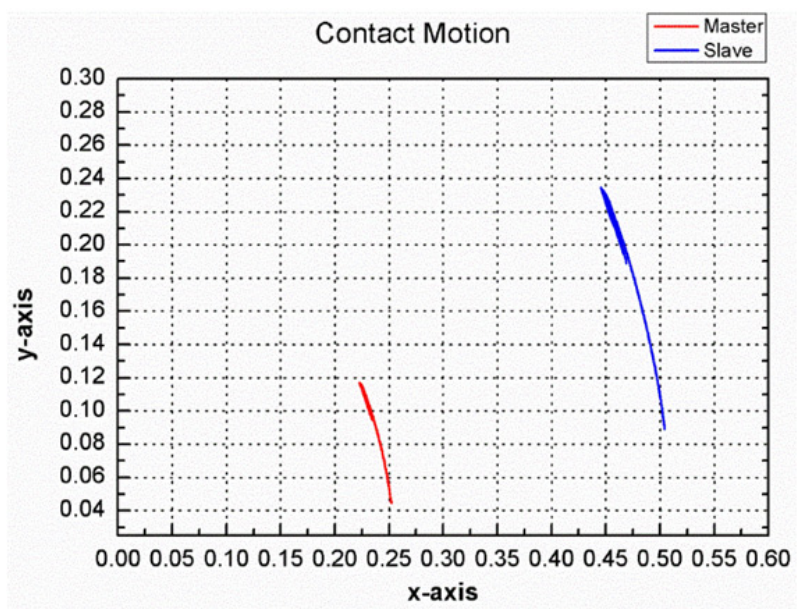

Figure 11. XY trajectory response during contact motion (soft object) 
During contact with the aluminium, the force for both $x$ and $y$ position tracked at slave system is twice larger than master manipulator. This is due to the virtual mass at slave system which is twice larger than master system. Overall, the law of action and reaction are achieved between master and slave system as shown in Figure 8 .

The $x$ and $y$ position response from slave manipulator are twice longer than the master manipulator for both $x$ and $y$ position as shown in Figure 8, Figure 9 shows the position response of XY trajectory for both master and slave manipulator during random contact motion, where the slave manipulator has virtually twice operation range compared to master manipulator. Again, the position response from both master and slave system are tracked perfectly as the trajectory of both master and slave system are the same.

Next, during contact with the sponge, the force for both $x$ and $y$ position tracked at slave system is twice larger than master manipulator. This is due to the virtual mass at slave system which is twice larger than master system. Overall, the law of action and reaction are achieved between master and slave system as shown in Figure 10.

Figure 10 shows that the $x$ and $y$ position response from slave manipulator are twice longer than the master manipulator for both $x$ and $y$ position. Meanwhile, Figure 11 shows the position response of XY trajectory for both master and slave manipulator during random contact motion, where the slave manipulator has virtually twice operational range compared to master manipulator. Again, the position response from both master and slave system are tracked perfectly as the trajectory of both master and slave system are the same.

Meanwhile, when the human operator released force at the master manipulator, the sponge eventually expanded to regain its shape as much as possible. During the expansion of sponge, an expansion force from the sponge pushed the slave manipulator which is in contact with sponge, pushing the master manipulator too, causing changes in position response in both master and slave manipulator, especially in $y$ axis direction, as can be observed in Figure 10 and Figure 11 Moreover, the human operator feels half of the expansion force exerted by the sponge from the master manipulator because it is scaled down by the scaling gain.

By observing the force response shown in Figure 8 and Figure 10, the force for $y$ axis at master and slave are higher compared to $x$ axis. This is because the human operator applied more force at $y$ axis during the contact motion. This also can be shown in position response where $y$ position deviated during the contact motion. Figure 9 and Figure 11 showed the trajectory motion where master and slave manipulator start from initial position and contact with the aluminium and sponge, respectively. In brief, human operator manipulated the master manipulator more in the $y$ axis direction.

\section{Discussion}

During the experiments on this micro-macro bilateral teleoperation control system with free motion, the human operator manipulated the master manipulator, the slave manipulator followed perfectly as shown in the XY trajectory response of both master and slave system. Moreover, the human operator operated the master manipulator with ease as there is very low frictional force produced by the gear. This is because the gear reduction ratio at master manipulator is lower. Thus, human operator was able to perform free motion comfortably and easily. Overall, the human operator can operate a larger scale manipulator by using a smaller scale manipulator.

During contact motion, human operator applied force to the master manipulator, slave manipulator applied the action force twice larger. Then, the reaction force exerted by the object to the slave manipulator, human operator received half of the magnitude of the reaction force from the master manipulator. This shows that the law of action and reaction achieved with scaling effect between micro-macro bilateral teleoperation control system. The stable contact of the slave manipulator is achieved on aluminium and sponge. The human operator can feel sharpness of reaction force from hard object. The friction force is almost neglected as there is no free motion during the contact motion of hard object. Overall, the reproducibility during contact motion is confirmed by these experiments.

However, during contact with the sponge, there is extra operational force to compress the sponge which is frictional force from gear. Then the expansion force need to overcome the frictional force at the slave system for the sponge to expand. Thus, human operator from master manipulator felt less expansion force from the sponge. Overall, the position tracking of the end-effector and the "law of action and reaction" are achieved between master and slave system in the workspace. This shows that the geared DC-motor was able to perform well in contact motion and reproduce the reaction force from the environment and the bilateral teleoperation control system is achieved. The validity of the standardization of modal space by ratio is achieved by the experimental results. 


\section{Conclusion}

The proposed MDOF micro-macro bilateral teleoperation control system is demonstrated. The performance of the MDOF micro-macro bilateral teleoperation control system showed a similar result as the non-micro-macro, except that the scaling of position and force responses at the end effector of both master and slave system are achieved. The position and force response were transmitted bilaterally between master and slave system. The scaling for force and position between micro master system and macro slave system were achieved by $\alpha$ and $\beta$ scaling gain, respectively. The experiments were conducted to validate the bilateral control system with the two link geared-manipulator. The haptic information which is the position and torque information from both master and slave manipulators were plotted and compared. Experimental results supported the theoretical framework of the proposed method and displayed accurate position and force tracking between micro master and macro slave system the standardized matrix was able to harmonize the standard of micro master system with the standard of macro slave system in the modal space. The proposed method called standardization of modal space ratio for MDOF micro-macro bilateral teleoperation control system was achieved and is promising for future micro-macro bilateral teleoperation applications in the industry.

All in all, the human operator was not just able to control a macro slave manipulator by operating a micro master manipulator, the human operator also able to aware of the environment at the macro slave manipulator. For instance, the human operator was able to feel the environment and halt the operation when the macro slave manipulator touches or hits an object. Henceforth, this would avoid any damage to the manipulator or the object. Moreover, this system is safer, accessible and human friendly in the automation industry, same as other existing teleoperation control system. Thus, standardization of modal space by ratio will be useful in fundamental personal support technology that will be developed for the future.

As for future work, there exists high operational force from the planetary gear in the form of frictional force. This brings operational force during the free motion. Moreover, high gear reduction ratio causes human operator to feel high stiffness during the operation. Thus compensation of frictional force is vital. Nevertheless, elimination/reduction of the backlash effect of the gear also must be done. This two to do list are to eliminate the characteristic of gear in order to obtain real haptic.

Additionally, the singularity is a common issue in MDOF manipulator. It gives a limitation and precaution for a MDOF manipulator during operation, or even injury or damage to the human operator or the manipulator itself. Thus, methods in eliminate singularity is a priority in MDOF manipulator to fully utilise the structure without compromising the MDOF bilateral teleoperation control system.

\section{Acknowledgments}

Identify The Authors wish to express their thanks to the Universiti Teknikal Malaysia Melaka (UTeM) under the UTeM ZAMALAH scheme, UTeM Short Term Grant (PJP/2O13/FKE(5D)/S01171) and (PJP/2015/FKE(1D)/S01392).

\section{References}

Hace, A., \& Jezernik, K. (2010). Bilateral teleoperation by sliding mode control and reaction force observer. Proc. IEEE Int. Symp. Ind. Electron, 1809-1816. http://dx.doi.org/10.1109/ISIE.2010.5637717

Iida, W., \& Ohnishi, K. (2004). Reproducibility and operationality in bilateral teleoperation. 8th IEEE International Workshop AMC, pp. 217-222. http://dx.doi.org/10.1109/AMC.2004.1297669

Kaneko, K. et al. (1998). Macro-micro bilateral teleoperation based on operational force feedforward. IEEE/RSJ International Conference on Intelligent Robots and Systems, pp. 1761-1769, October, 1998. http://dx.doi.org/10.1109/IROS.1998.724852

Katsura, S., Matsumoto, Y., \& Ohnishi, K. (2007). Modeling of force sensing and validation of disturbance observer for force control. 29th Annual Conference of the IEEE Industrial Electronics Society, IECON '03, 54(1), 530-538. http://dx.doi.org/10.1109/TIE.2006.885459

Lawrence, D. A. (1993). Stability and transparency in bilateral teleoperation. IEEE Transactions on Robotics and Automations, 9(5), 624-637. http://dx.doi.org/10.1109/AMC.2010.5464046

Motoi, N. et al. (2010). Bilateral Control with Different Inertia Based on Modal Decomposition. 11th IEEE International Workshop on Advanced Motion Control, AMC, pp. 697-702. http://dx.doi.org/10.1109/AMC.2010.5464046

Murakami, T., \& Ohnishi, K. (1993). Torque sensorless control in multidegree-of-freedom manipulator. IEEE Transactions on Industrial Electronics, 40(2), 259-265. http://dx.doi.org/10.1109/41.222648 
Ohnishi, K., Matsui, N., \& Hori, Y. (1994). Estimation, identification, and sensorless control in motion control System. IEEE, 82(8), 1253 - 1265. http://dx.doi.org/10.1109/5.301687

Ohnishi, K., Shibata, M., \& Murakami, T. (1996). Motion control for advanced mechatronics. IEEE/ASME Transactions of Mechatronics, 1(1), 56-67. http://dx.doi.org/10.1109/3516.491410

Salcudean, S. E., \& Yan, J. (1994). Towards a force-reflecting motion-scaling system for microsurgery. ICRA Procedings of the IEEE International Conference on Robotics and Automation, 3, pp. 2296-2301. http://dx.doi.org/10.1109/ROBOT.1994.350943

Sano, A., Fujimoto, H., \& Takai, T. (2000). Personal scaling on micro-teleoperation. 26th IEEE Anual Conference of the IEEE Industrial ELectronics Society, IECON '00, 1, pp. 7-12. http://dx.doi.org/10.1109/IECON.2000.973118

Shimono, T., \& Ohnishi, K. (2007). Reproduction of real world force sensation by micro-macro bilateral control with respect to standardized modal space. IECON Proceedings (Industrial Electronics Conference), pp. 374-379. http://dx.doi.org/10.1109/IECON.2007.4460092

Susa, S. et al. (2007). Transmission of force sensation by micro-macro bilateral control with respect to standardized model space. International Conference on Mechatronics, 1(May), pp. 1-6. http://dx.doi.org/10.1109/ICMECH.2007.4280061

Susa, S., \& Shimono, T., et al. (2008). Transmission of force sensation by micro-macro bilateral control with scaling of control gains. International Workshop on Advanced Motion Control, AMC, 1, pp.532-537. http://dx.doi.org/10.1109/AMC.2008.4516123

Susa, S., Natori, K., \& Ohnishi, K. (2008). Three-channel micro-macro bilateral control system with scaling of control gains. 34th Annual Conference of the IEEE Industrial Electronics Society, IECON, pp. 2598-2603, 2008. http://dx.doi.org/10.1109/IECON.2008.4758366

Takeo, K., \& Kosuge, K. (1997). Implementation of the micro-macro teleoperation system without using slave-side force sensors. International Conference on Robotics and Automation, 2(April), pp. 1600-1605. http://dx.doi.org/10.1109/ROBOT.1997.614369

Wei, L. J., Shukor, A. Z. H., \& Jamaluddin, M. H. (2016). Investigation on the effects of outer-loop gains, inner-loop gains and variation of parameters on bilateral teleoperation control system using geared DC-motor. International Journal of Mechanical \& Mechatronics Engineering IJMME-IJENS, 16(01), 54 69. Retrieved from http://ijens.org/Vol_16_I_01/161601-2929-IJMME-IJENS.pdf

Wei, L. J., Shukor, A. Z. H., \& Jamaluddin, M. H. (2015). Workspace control of two link planar robot using Micro-Box 2000. Journal Teknologi, 77(20), 9-18. http://dx.doi.org/10.11113/jt.v77.6545

\section{Copyrights}

Copyright for this article is retained by the author(s), with first publication rights granted to the journal.

This is an open-access article distributed under the terms and conditions of the Creative Commons Attribution license (http://creativecommons.org/licenses/by/4.0/). 